\title{
A Broadband Transimpedance Amplifier with Optimum Bias Network
}

\author{
Qian $\mathrm{Gao}^{1, a}$, Sheng $\mathrm{Xie}^{1, \mathrm{~b}^{*}}$, Luhong Mao ${ }^{1, \mathrm{c}}$ and Sicong $\mathrm{Wu}^{1, \mathrm{~d}}$ \\ ${ }^{1}$ School of Electronic and Information Engineering, Tianjin University, Tianjin 300072, China

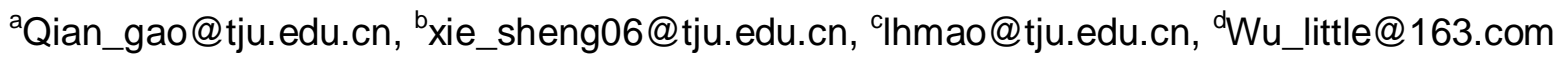 \\ *The corresponding author
}

\begin{abstract}
Keywords: Regulated cascode; Transimpedance amplifier; Matching networks; CMOS
\end{abstract}
\begin{abstract}
A novel transimpedance amplifier (TIA) based on regulated cascode (RGC) configuration was proposed, in which a bias network was added to ensure the main amplifier and the auxiliary amplifier of RGC operate at optimum condition. To achieve high bandwidth, a T-passive matching network was introduced at the input. Since both amplifiers have optimum gain-bandwidth product (GBW), thus low noise and high bandwidth can be obtained in our design scheme. The proposed TIA was designed based on UMC $0.18 \mu \mathrm{m}$ CMOS process, and the simulation results demonstrated that our TIA has a transimpedance gain of $51 \mathrm{~dB} \Omega$ and a $-3 \mathrm{~dB}$ bandwidth of $14 \mathrm{GHz}$ with a input capacitance of $0.3 \mathrm{pF}$. The average equivalent input noise current spectral density is about 13 $\mathrm{pA} / \sqrt{\mathrm{Hz}}$ within $-3 \mathrm{~dB}$ bandwidth.
\end{abstract}

\section{Introduction}

With the rapid development of mobile Internet, cloud computing and Internet of Things, high speed data communication between board-to-board, chip-to-chip and on-chip is required [1], and thus make the optical interconnection become a hot issue in recent years. On the other hand, with the continuous scaling of semiconductor technology, the peak transit frequency of the deep submicron MOSFET device has exceeded tens of gigahertz. Due to the cost-effective performance with a friendly integration scheme, high speed optoelectronic integrated circuits (OEICs) based on CMOS technology have been widely explored [2-5].

As a key block of optical transceiver, the transimpedance amplifier (TIA) determines the overall performance of the receive chain, such as operation speed and sensitivity. As a result, improving the bandwidth of TIA becomes one of the critical steps to achieve high speed optical receiver front-end. Due to the low impedance of regulated cascode (RGC) configuration [3], which can neutralize the effects of large photodiode (PD) capacitance and the parasitic capacitance, RGC topology has been widely employed in gigabit optical receiver. Additionally, the RGC TIA also has a lower input referred noise current with respect to common gate (CG) amplifier. To obtain higher bandwidth, various bandwidth enhancement techniques have been demonstrated in RGC configuration, such as auxiliary amplifier improvement technique [4, 5], passive matching network [6-13] and polezero cancellation $[14,15]$. Compared with other techniques, the passive matching network is found more effective both in bandwidth enhancement and noise reduction.

This paper proposed a novel TIA circuit based on RGC configuration, in which an optimum bias (OB) was added to improve the gain-bandwidth product (GBW), and a T-passive matching network (PMN) was introduced to further extend the bandwidth and reduce the input-referred noise current spectral density.

\section{The Novel TIA with Optimum Bias}

As shown in Fig. 1, conventional RGC configuration is constructed by a common-source (CS) auxiliary amplifier $\left(M_{2}\right.$ and $\left.R_{\mathrm{B}}\right)$ and a common-gate $(\mathrm{CG})$ main amplifier $\left(\left(M_{1}\right.\right.$ and $\left.R_{\mathrm{D}}\right)$, and the auxiliary amplifier is connected between the gate of $M_{1}$ and node X. Where the PD is electrically modeled by a current source $I_{\mathrm{PD}}$ shunted with a junction capacitance $C_{\mathrm{PD}}$. To achieve better 
performance parameters, the main amplifier is preferably selected an optimum DC bias at the expense of decreasing the gain-bandwidth product (GBW) of auxiliary amplifier.

To solve this problem and obtain maximal GBW, an optimum bias network consisting of $C, R_{\mathrm{B} 1}$ and $R_{\mathrm{B} 2}$, marked by the dotted line box, is added to the conventional RGC configuration, as shown in Fig. 2. Where the voltage divider formed by $R_{\mathrm{B} 1}$ and $R_{\mathrm{B} 2}$ is utilized to provide an optimum bias for $M_{1}$, and the capacitance $C$ is used to isolate the DC biases of both amplifiers and pass the AC signal. Since the DC operating points of both amplifiers are independent of each other, they can be set respectively to the optimum bias condition, and thus a larger GBW can be realized. In our design, the values of resistances $\left(R_{\mathrm{B} 1} \& R_{\mathrm{B} 2}\right)$ should be large enough to prevent the $\mathrm{AC}$ signal shunting to ground, and avoid extra noise introduced by these resistances.

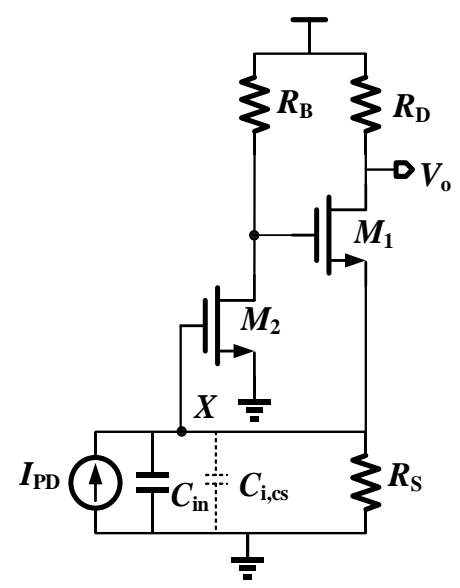

Figure 1. Schematic of conventional RGC

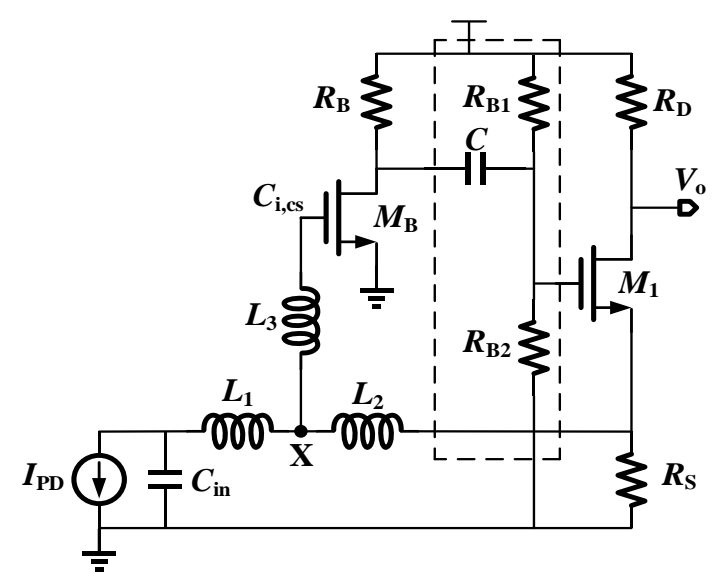

Figure 2. The proposed RGC with optimal bias

As shown in Fig. 1, the capacitances $C_{\mathrm{in}}, C_{\mathrm{i}, \mathrm{cs}}$ and $C_{\mathrm{gs} 1}$ are shunted at the input node, and they form a large capacitance that would greatly degrade the RGC performance at high frequency. Where $C_{\text {in }}$ is the total input capacitance including PD capacitance, $C_{\mathrm{gs} 1}$ is the gate-source capacitance of $M_{1}$, $C_{\mathrm{i}, \mathrm{cs}}$ is the total parasitic capacitance of CS amplifier, and it can be expressed as

$$
C_{\mathrm{i}, \mathrm{CS}}=\boldsymbol{C}_{\mathrm{gsB}}+\left(1+g_{\mathrm{mB}} R_{\mathrm{B}}\right) \boldsymbol{C}_{\mathrm{gdB}}
$$

where $\mathrm{C}_{\mathrm{gsB}}$ and $\mathrm{C}_{\mathrm{gdB}}$ are respectively the gate-source and the gate-drain capacitance of $M_{\mathrm{B}}, \mathrm{g}_{\mathrm{mB}}$ is the transconductance of $M_{\mathrm{B}}$.

In broadband circuit design, the passive matching network was usually used to alleviate the gain-bandwidth limitation. Ref [10] introduced a matching network formed by the inductors of $L_{1}$ and $L_{2}$ to reduce the effect of shunt capacitances, and a fifth ladder network was constructed at the input to achieve maximum bandwidth enhancement and maximum gain flatness [10]. However, the photocurrent would flow through the input parasitic capacitance $C_{\mathrm{i}, \mathrm{cs}}$ rather than RGC input impedance at high frequencies, thus leads to the reduction of RGC bandwidth. To avoid this situation, the inductor $L_{3}$ is therefore inserted between the $X$ node and the gate of $M_{\mathrm{B}}$ [8], as shown in Fig. 2. The relationship between the drain current of $M_{\mathrm{B}}$ and $V_{x}$ is

$$
G_{\mathrm{mB}}=\frac{i_{\mathrm{d}, \mathrm{MB}}}{V_{\mathrm{x}}}=\frac{g_{\mathrm{mB}}}{1-\omega^{2} L_{3} C_{\mathrm{i}, \mathrm{CS}}} .
$$

As can be seen in Eq.2, the effective conductance $G_{\mathrm{mB}}$ increases with increasing the frequency, which compensates the gain reduction caused by the Miller effect of CS amplifier at high frequencies. By using T-passive matching network and optimum bias, the GBW of proposed TIA is up to its theoretical limit. 


\section{Noise Analysis and Reduction}

As we know, the noise performance of input stage directly affects the bit error rate (BER) of whole optical receiver. Therefore, the noise of RGC input stage will be analyzed in the following by using the standard noise analysis in terms of $E_{\mathrm{n}}-I_{\mathrm{n}}$ model [9]. The equivalent input noise current of proposed TIA is given by

$$
\overline{i_{\mathrm{n}, \mathrm{eq}}^{2}} \cong\left(1-\omega^{2} L_{1} C_{\mathrm{in}}\right)^{2} \cdot\left(N_{\mathrm{b}}^{2}+N_{\mathrm{T}}^{2}\right)+\omega^{2} C_{\mathrm{in}}^{2}\left(N_{1}^{2}+N_{\mathrm{B}}^{2}\right)
$$

where

$$
\begin{aligned}
& N_{\mathrm{b}}^{2}=\left(1-\omega^{2}\left(\frac{L_{2}}{\left(1+G_{\mathrm{mB}} R_{\mathrm{B}}\right)} C_{\mathrm{i}, \mathrm{CS}}\right)\right)^{2}\left(\omega^{2}\left(\left(1+G_{\mathrm{mB}} R_{\mathrm{B}}\right) C_{\mathrm{gs} 1}\right)^{2} N_{1}^{2}+\omega^{2} C_{\mathrm{gsB}}^{2} N_{\mathrm{B}}^{2}+N_{\mathrm{R}}^{2}\right) \\
& N_{\mathrm{T}}^{2}=\omega^{2} C_{\mathrm{gsB}}^{2} N_{\mathrm{B}}^{2}+\omega^{2} C_{\mathrm{i}, \mathrm{CS}}^{2} N_{1}^{2} \\
& N_{\mathrm{R}}^{2}=\frac{4 k T}{R_{\mathrm{D}}}+\frac{4 k T}{R_{\mathrm{S}}}+\frac{4 k T\left(\gamma G_{\mathrm{mB}}+1 / R_{\mathrm{B}}\right)}{\left(G_{\mathrm{mB}}+1 / R_{\mathrm{B}}\right)^{2}} \frac{1}{R_{\mathrm{s}}^{2}} \\
& N_{\mathrm{B}}^{2}=\frac{4 k T\left(\gamma G_{\mathrm{mB}}+1 / R_{\mathrm{B}}\right)}{\left(G_{\mathrm{mB}}+1 / R_{\mathrm{B}}\right)^{2}} \\
& N_{1}^{2}=\frac{4 k T\left(\gamma g_{\mathrm{m} 1}+1 / R_{\mathrm{D}}\right)}{g_{\mathrm{m} 1}^{2}\left(1+G_{\mathrm{mB}} R_{\mathrm{B}}\right)^{2}}
\end{aligned}
$$

where $k$ is Boltzmann constant, and $T$ is the absolute temperature.

As it can be seen from Eq. 3, the noise from $R_{\mathrm{D}}$ and $R_{\mathrm{S}}$ is dominant at low frequencies. In order to reduce the low frequency noise, the value of $R_{\mathrm{S}}$ should be increased, but this will lower the drain voltage of $M_{\mathrm{B}}$ in conventional RGC, which make the main amplifier deviate the optimum bias condition. On the other hand, if we reduce the value of $R_{\mathrm{B}}$ or/and the gate width of $M_{\mathrm{B}}$, the auxiliary amplifier gain will decrease, thus increasing the channel thermal noise contribution from $M_{\mathrm{B}}$. Fortunately, the optimum bias network added in proposed TIA guarantees both amplifiers can operate at respective optimum bias condition while increasing the value of $R_{\mathrm{s}}$.

According to the second term of Eq. 3, the growth rate of input noise current at high frequencies depends on the input capacitance $C_{\text {in. }}$. The matching network introduced in Fig. 2 isolates the effects of parasitic capacitance, and reduces the noise contribution from $M_{1}$ and $M_{\mathrm{B}}$ at the frequency of desired bandwidth. Therefore, the input noise current decreases significantly at high frequencies with respect to the conventional RGC configuration.

\section{Simulation Results}

The proposed TIA is simulated in UMC $0.18 \mu \mathrm{m}$ CMOS process, and a PD capacitance of $0.3 \mathrm{pF}$ is adopted. The frequency response with/without the optimum bias (OB) and T-passive matching network $(\mathrm{PMN})$ is illustrated in Fig. 3. For comparison, the frequency response of conventional $\mathrm{RGC}$ is also shown. As can be seen, the $-3 \mathrm{~dB}$ bandwidth expands from 7 to $8.2 \mathrm{GHz}$ after introduction of $\mathrm{OB}$, and it increases to $14 \mathrm{GHz}$ by using $\mathrm{OB}$ and $\mathrm{PMN}$.

Fig. 4 shows the input referred noise current spectrum density of RGC with/without $\mathrm{OB}$ and PMN. The noise of TIA with OB is less than that of the conventional RGC, but the noise of TIA with OB and PMN decreases significantly at high frequencies, this is well agree with the analysis of Section 3. The average equivalent input noise current spectral density is about $13 \mathrm{pA} / \sqrt{\mathrm{Hz}}$ within -3 $\mathrm{dB}$ bandwidth. 
Fig. 5 shows the simulated eye diagram with a $2^{31}-1$ pseudo-random bit sequence (PRBS) for 10 $\mathrm{Gb} / \mathrm{s}$ and $15 \mathrm{~Gb} / \mathrm{s}$, and the photocurrent amplitude is $50 \mu \mathrm{A}$. The eye diagram indicates that the increase of bandwidth and the decrease of noise are at the cost of ringing response in the time domain. Such a response results from the zeros and poles added to the transfer function by establishing matching network at the input node. This in turn partly causes the inter-symbol interference and closes vertically eye, but our proposed TIA still achieves high performance.

For comparison, Table 1 summarized the performance of our proposed TIA and the results of prior works. Obviously, the proposed TIA has larger bandwidth and smaller noise while keeping other parameters comparable.

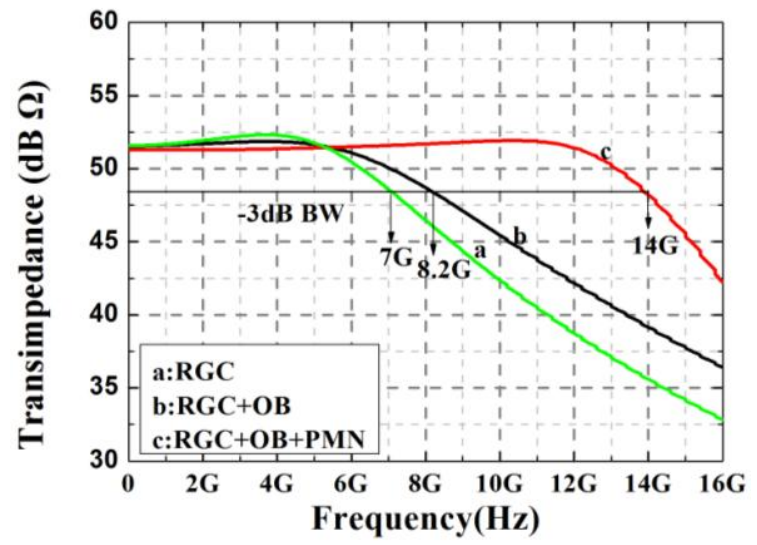

Figure 3. Frequency response of RGC with/ without $\mathrm{OB}$ and PMN

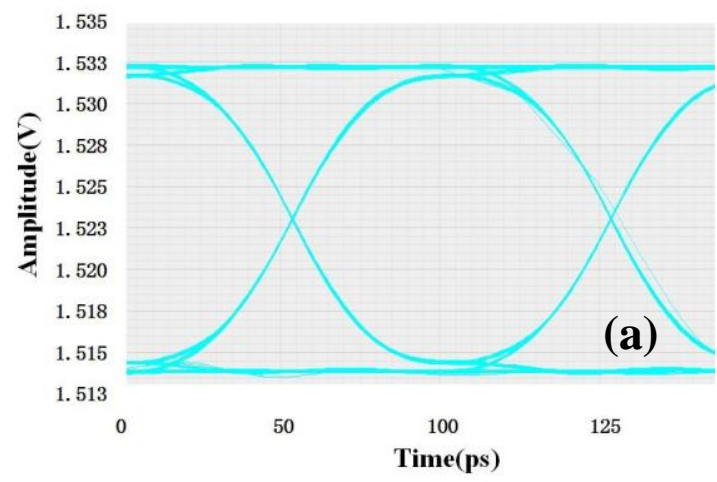

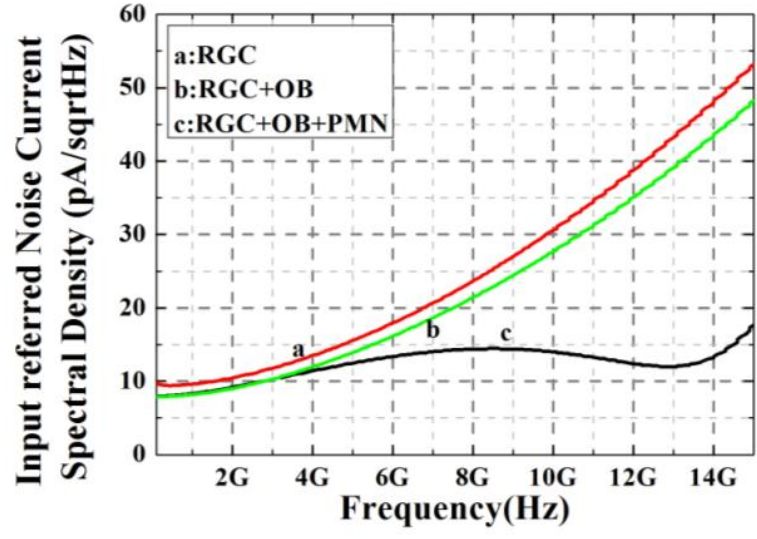

Figure 4. Input referred noise current spectrum density of RGC with/without OB and PMN

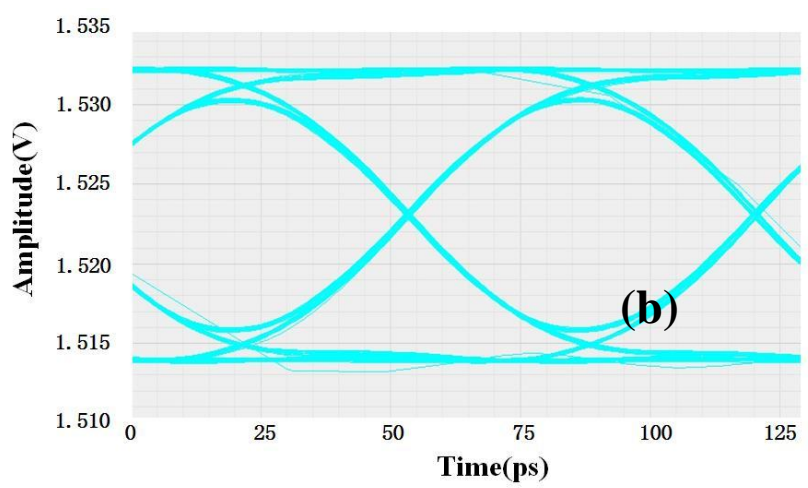

Figure 5. Simulated eye diagram of the proposed TIA at (a) $10-\mathrm{Gb} / \mathrm{s}$ and (b) $15-\mathrm{Gb} / \mathrm{s}$.

Table 1 Summary and comparison of other works.

\begin{tabular}{cccccc}
\hline Parameter & Ref. [8] & Ref. [9] & Ref. [11] & Ref. [12] & This work \\
\hline $\begin{array}{c}\text { Transimpedance } \\
\text { Gain }(\mathrm{dB} \Omega)\end{array}$ & 57 & 53 & 54 & 59 & 51 \\
$\begin{array}{c}\text { Bandwidth } \\
\quad(\mathrm{GHz})\end{array}$ & 8.2 & 8 & 7 & 8.6 & 14 \\
$\begin{array}{c}\text { Input PD } \\
\text { capacitance (pF) } \\
\text { Input Noise } \\
\quad(\mathrm{pA} / \sqrt{\mathrm{H} z})\end{array}$ & 0.3 & 0.25 & 0.3 & 0.15 & 0.3 \\
$\begin{array}{c}\text { Number of } \\
\text { inductance }\end{array}$ & 3 & 18 & 18 & 25 & 13 \\
\hline
\end{tabular}




\section{Conclusion}

In this paper, a novel broadband TIA is proposed based on RGC configuration, in which the optimum bias and T-passive matching network are introduced to neutralize the effects of parasitic capacitance. Simulation results shown that both the optimum bias and the passive matching network can effectively expand the bandwidth and reduce the noise. Since the eye diagram is clear at 15 $\mathrm{Gb} / \mathrm{s}$, our proposed TIA is expected to be applied for high speed optical interconnection.

\section{Acknowledgments}

This work is supported by the National Natural Science Foundation of China (No. 61474081).

\section{References}

[1] J. Kim and J. F. Buckwalter, A 40-Gb/s optical transceiver front-end in $45 \mathrm{~nm}$ SOI CMOS, IEEE J. of Solid-State Circuits. 47 (2012) 615-626.

[2] S. Xie, X. Z. Tao, L. H. Mao, et al. High-Gm differential regulated cascode transimpedance amplifier, Trans. Tianjin Univ. 22 (2016) 345-351.

[3] S.M. Park, H.J. Yoo, 1.25-Gb/s regulated cascode CMOS transimpedance amplifier for gigabit ethernet applications, IEEE J. Solid-State Circuits 39 (2004) 112-121.

[4] L. Chen, P.A. Samuel, Low-power, $26 \mathrm{GHz}$ transformer-based regulated cascode transimpedance amplifier in $0.25 \mu \mathrm{m}$ SiGe BiCMOS, Bipolar/BiCMOS Circuits and Technology Meeting, ( 2011) 819-825.

[5] Z.D. Yun, A high-speed fully-integrated POF receiver with large-area photo detectors in $65 \mathrm{~nm}$ CMOS, IEEE J. Solid-State Circuits. 47 (2012) 2080-2092.

[6] L.L. Chen, Z.Q. Li, Z.G. Wang, A 10-Gb/s OEIC with Meshed Spatially-Modulated Photo Detector in 0.18- $\mu \mathrm{m}$ CMOS Technology, IEEE J. of Solid-State Circuits, 46 (2011) 1158-1167.

[7] D. Li et al. A Low-noise design technique for high-speed CMOS optical receivers, IEEE J. of Solid-State Circuits, 49 (2014) 1437-1447.

[8] M. Seifouri, P. Amiri, M. Rakidei, Design of broadband transimpedance amplifier for optical communication systems, micreoelectronics Journal, 46 (2015) 679-684.

[9] Q.W. Song, L.H. Mao, S. Xie, et al. Novel pre-equalization transimpedance amplifier for 10 $\mathrm{Gb} / \mathrm{s}$ optical interconnects, Journal of Semiconductors, 36 (2015) 1-5.

[10]Z. Lu, K.S. Yeo, J.G. Ma, et al. Broad-band technique for transimpedance amplifiers, IEEE Trans. Circuits Syst. 54 (2007) 590-599.

[11]B. Analui, A. Hajimiri, Bandwidth enhancement for transimpedance amplifiers, IEEE J. Solid-State Circuits 39 (2004) 1263-1270.

[12]Z.H. Lu, K.S. Yeo, W.M. Lim, et al. Design of a CMOS broadband transimpedance amplifiers with active feedback, IEEE Trans. Very Large Scale Integ. (VLSI) Syst. 18 (2010) 461-471.

[13]C.Y. Wang, C.S. Wang, C.K. Wang, An $18 \mathrm{~mW}$ two stage CMOS transimpedance amplifier for $10 \mathrm{~Gb} / \mathrm{s}$ optical application. IEEE Asian Solid State Circuits Conference, 2007.

[14]A.H.M. Shirazi, R. Molavi, P.S. Woo, et al. A low-power DC to $27 \mathrm{GHz}$ transimpedance amplifier in $0.13 \mu \mathrm{m}$ CMOS using inductive-peaking and current-reuse techniques, International Midwest Symposium on Circuits and Systems, (2014) 961-964.

[15]B. Samira, P. Calvin, A. Jorge, et al. A $40 \mathrm{~Gb} / \mathrm{s}$ Transimpedance Amplifier in $65 \mathrm{~nm}$ CMOS, Proceedings of 2010 IEEE International Symposium on Circuits and Systems (ISCAS), (2010) 757-760. 